Meta

Journal des traducteurs

Translators' Journal

\title{
Le traducteur dans un théâtre à mille temps
}

\section{Agata Gołębiewska}

Volume 62, numéro 3, décembre 2017

La traduction littéraire comme création

URI : https://id.erudit.org/iderudit/1043952ar

DOI : https://doi.org/10.7202/1043952ar

Aller au sommaire du numéro

Éditeur(s)

Les Presses de l’Université de Montréal

ISSN

0026-0452 (imprimé)

1492-1421 (numérique)

Découvrir la revue

Citer cet article

Gołębiewska, A. (2017). Le traducteur dans un théâtre à mille temps. Meta, 62(3), 614-623. https://doi.org/10.7202/1043952ar

\section{Résumé de l'article}

Le rôle du traducteur dans le domaine du théâtre est la conséquence directe de la position qu'y occupe le texte. Le théâtre contemporain occidental ayant de moins en moins pour mission de donner une représentation fidèle d'un texte source, et cherchant plutôt à créer une expérience collective, la traduction s'y inscrit, comme processus de création, dans la totalité de la démarche théâtrale. Cette évolution brouille encore davantage la frontière entre traduction et écriture, ce qui soulève de nombreuses questions, mais ouvre, en même temps, des espaces inattendus à la créativité. Dans le théâtre contemporain expérimental, il est coutumier de prendre des libertés avec une oeuvre de littérature dramatique (la seule limite étant celle de la loi, donc de l'accord nécessaire des auteurs ou des ayants droit s'ils existent). Le traducteur qui participe à un projet de spectacle travaille étroitement avec le metteur en scène et le dramaturge. Il doit, au-delà de ses compétences linguistiques, bien connaître les spécificités d'un travail sur scène et comprendre notamment qu'il y est problématique de parler d'un résultat non évolutif. Une expérience de théâtre et des affinités avec les collaborateurs impliqués sont indispensables dans ce processus, dont l'issue est une forme vivante, une expérience créatrice collective.
Ce document est protégé par la loi sur le droit d'auteur. L’utilisation des services d’Érudit (y compris la reproduction) est assujettie à sa politique d'utilisation que vous pouvez consulter en ligne.

https://apropos.erudit.org/fr/usagers/politique-dutilisation/ 


\title{
Le traducteur dans un théâtre à mille temps
}

\author{
AGATA GOEĘBIEWSKA \\ Traductrice littéraire, Paris, France \\ agatag@wanadoo.fr
}

\section{RÉSUMÉ}

Le rôle du traducteur dans le domaine du théâtre est la conséquence directe de la position qu'y occupe le texte. Le théâtre contemporain occidental ayant de moins en moins pour mission de donner une représentation fidèle d'un texte source, et cherchant plutôt à créer une expérience collective, la traduction s'y inscrit, comme processus de création, dans la totalité de la démarche théâtrale. Cette évolution brouille encore davantage la frontière entre traduction et écriture, ce qui soulève de nombreuses questions, mais ouvre, en même temps, des espaces inattendus à la créativité. Dans le théâtre contemporain expérimental, il est coutumier de prendre des libertés avec une œuvre de littérature dramatique (la seule limite étant celle de la loi, donc de l'accord nécessaire des auteurs ou des ayants droit s'ils existent). Le traducteur qui participe à un projet de spectacle travaille étroitement avec le metteur en scène et le dramaturge. Il doit, au-delà de ses compétences linguistiques, bien connaître les spécificités d'un travail sur scène et comprendre notamment qu'il y est problématique de parler d'un résultat non évolutif. Une expérience de théâtre et des affinités avec les collaborateurs impliqués sont indispensables dans ce processus, dont l'issue est une forme vivante, une expérience créatrice collective.

\section{ABSTRACT}

The role of the translator in theater is the direct result of the very role given to the text itself. Today, contemporary Western theater seeks to generate a shared experience rather than provide a faithful representation of a literary work. As a result, this evolution in theater blurs the frontier between translation and writing, a fact which not only raises numerous questions, but also opens unexpected spaces for creativity. In experimental contemporary theater it is customary to take some artistic licence with a work of dramatic literature (the only limit being that fixed by the law, i.e., the consent of the author(s) or copyright holders). The translator who takes part in a theatrical project works closely with both the director and dramaturg. Beyond his or her linguistic skills, he or she must be familiar with the specificities of the work on stage and understand how problematic it is to talk about a fixed performance. An experience in theater and affinities with the collaborators involved are essential in this process, the outcome of which is a living form, a collective experience of creation.

\section{RESUMEN}

El papel del traductor en el ámbito teatral es la consecuencia directa de la posición que ocupa en él el texto. El teatro contemporáneo occidental tiene cada vez menos la finalidad de ofrecer una representación fiel de un texto fuente y pretende más bien generar una experiencia colectiva. En este contexto, la traducción, como proceso creativo, forma parte del proceso teatral en su conjunto. Esta evolución difumina todavía más los límites entre traducción y escritura, lo cual produce numerosos planteamientos, a la vez que abre espacios inesperados para la creatividad. En el teatro contemporáneo experimental, es habitual tomarse la libertad de alejarse de una obra literaria dramática (el único límite es la ley, es decir, contar con la autorización necesaria de los autores o de sus derechohabientes, cuando estos existan). El traductor que participa en un proyecto de espectáculo trabaja en estrecha colaboración con el director teatral y con el autor. Además de 
sus competencias lingüísticas, debe conocer bien las especificidades de la labor escénica y comprender sobre todo que es difícil hablar de un resultado inamovible. En este proceso, una experiencia teatral y afinidades con las personas con las que trabajará son indispensables; el resultado es una forma viva, una experiencia de creación colectiva.

\section{MOTS CLÉS/KEYWORDS/PALABRAS CLAVE}

théâtre, traducteur, expérience collective, création theater, translator, collective experience, creation teatro, traductor, experiencia colectiva, creación

Si, au sein du monde du théâtre occidental issu de la tradition aristotélicienne, la traduction a toujours eu pour fondement la transmission d'un texte source, elle se trouve aujourd'hui englobée comme processus de création dans la totalité de la démarche théâtrale. En effet, nous allons voir comment, dans ce domaine, la traduction sort du cadre de l'interprétation pour participer à un discours qui est potentiellement un texte en devenir dont la finalité est une véritable expérience de création. Poussant cette expérience jusqu'à ses ultimes limites, une tendance du théâtre contemporain semble mettre un terme à la séparation des rôles instaurée depuis quelques siècles afin de faire œuvre commune permettant à chaque intervenant, qu'il soit acteur, auteur ou traducteur, d'être reconnu dans sa dimension créatrice.

\section{1. Évolution du théâtre d'aujourd'hui}

On ne peut parler de la traduction théâtrale sans évoquer l'évolution qu'a connue le théâtre durant ces dernières décennies. Depuis longtemps déjà, la mise en scène est «considérée comme une pratique artistique spécifique, comme une écriture scénique qui n'a point à être commandée par la logique du texte écrit», pour citer Hans-Thies Lehmann (2010/2006: 9). «La ligne de partage entre texte et scène, longtemps critère définitoire du théâtre, se déplace et se reconfigure sur les scènes actuelles», dit, plus récemment encore, Rafaëlle Jolivet Pignon (2015: 17). Le sens de tous ces efforts est de sortir du schéma consistant à présenter une œuvre figée (le texte, comme la mise en scène). Le grand nombre de spectacles interactifs, itinérants, d'improvisations ou de works in progress en témoignent, et signalent surtout le développement de nouveaux langages scéniques. En effet, les notions mêmes de spectateur ou de spectacle correspondent de moins en moins à la réalité du théâtre d'aujourd'hui.

Henri Gouhier ${ }^{1}$ parlait d'un art à deux temps où la réalisation scénique constitue le deuxième temps, le premier étant celui de l'écriture. Il rendit compte des pratiques, notamment de celles de l'avant-garde américaine des années 1960, où les deux temps semblaient se fondre en un seul à force d'improvisation et de création collective, et où «création et exécution coïncidaient» (Danan 2015: 11). Pourtant, il en vint à constater, non sans raison, qu'il s'agissait souvent d'une modification du mode de production du texte sans que pour autant le modèle soit vraiment annulé. Pour sortir de cette opposition binaire, représentée d'une part par l'approche classique de Gouhier, et d'autre part par l'avant-garde des années 1960, ne conviendrait-il pas de se demander si, au lieu de fondre écriture et réalisation en un seul temps, le défi ne serait pas plutôt de trouver un prisme dans lequel le nombre des temps possibles deviendrait infini? «Formes multiples, temps multiples, processus faisant s'interpénétrer ces différents 
temps», proposait Joseph Danan (2015: 11) : finalement, n'est-ce pas ce qui rendrait tout un chacun acteur au sein du projet?

\section{La traduction comme dispositif créatif}

Si l'on devait citer l'élément le plus caractéristique du théâtre contemporain, ce serait très probablement la volonté de reconnaître le rôle créatif de chaque personne impliquée dans le spectacle: les membres de l'équipe artistique, tels que les comédiens, scénographes, créateurs lumière, créateurs son, etc., ainsi que les membres des équipes techniques, qui peuvent être, eux aussi, directement intégrés à la représentation, sans hiérarchie. Le signe le plus manifeste de la reconnaissance de ce rôle est l'apparition sur scène de toutes ces personnes pour saluer le public au côté des acteurs. Il arrive parfois que cette reconnaissance soit également matérielle, avec une rémunération égale pour tous. Il s'agit donc là d'une démocratisation du geste théâtral, aussi bien que d'une volonté du monde du théâtre de contribuer à la démocratisation de la société. La scène elle-même n'est plus délimitée par le seul plateau. Elle englobe maintenant les spectateurs. Jean-Michel Bruyère, du collectif marseillais LFK ${ }^{2}$ a pu dire, à l'occasion du colloque «Submission, Rage, Freedom», organisé à Nowy Teatr à Varsovie en avril 2016, qu'il ne croyait pas à la pertinence du professionnalisme dans l'art et notamment dans le théâtre. Selon lui, «la seule différence entre nous [qui créons un spectacle] et les spectateurs est que nous avons le droit d'utiliser la porte du personnel ${ }^{3}$ ». Un tel propos est évidemment extrême, très révélateur toutefois d'un certain esprit présent dans le théâtre contemporain, qui place la créativité et l'engagement bien avant la prouesse technique et la formation institutionnelle de l'ensemble des personnes impliquées dans la création théâtrale.

Ce théâtre, postdramatique, déconstruit la narration, ou l'idée même de personnages. Les acteurs y parlent souvent en leur nom propre: ils ne jouent plus un rôle mais s'expriment en tant que personnes et improvisent, de façon plus ou moins préparée. En outre, dans ce théâtre, l'auteur est souvent dramaturge - un terme à prendre dans sa dimension contemporaine, c'est-à-dire chargé par le metteur en scène du choix, de la collation ou de l'écriture de textes qui formeront la trame d'un spectacle. Il peut également modifier une traduction, en collaboration étroite avec les comédiens, le metteur en scène et le traducteur ou la traductrice. Dans cette tendance théâtrale, le texte s'écrit sur scène, pour un spectacle-projet particulier. Ainsi les reprises de pièces se font rares; puisqu'il s'agit, la plupart du temps, de commandes où un auteur écrit un texte lié à un contexte si particulier qu'il reste unique. Le texte n'est définitif qu'au tout dernier moment, car il évolue en lien étroit avec les répétitions, et même la première n'est plus une échéance mettant un terme au travail. Nous sommes donc bien loin de la situation traditionnelle, où le traducteur travaille à partir d'une pièce déjà publiée afin de produire un autre texte. D'ailleurs, même si une œuvre a déjà fait l'objet d'une traduction publiée, il n'est pas rare qu'un metteur en scène en commande une nouvelle, car il a besoin que la langue de la traduction corresponde parfaitement avec son idée de mise en scène. S'il le peut, il confie la traduction à une personne de confiance ou bien décide de traduire lui-même le texte source - ce qui pose la question de ses compétences linguistiques et des limites de l'adaptation. Le garde-fou à cette créativité reste, bien entendu, le code de la propriété intellectuelle. Par ailleurs, ce qui est important à comprendre, est le fait que dans le 
théâtre d'aujourd'hui il ne s'agit pas de servir un texte, mais de dialoguer avec lui. Le respect, l'estime pour un auteur ou une œuvre ne s'y mesurent pas à la fidélité avec laquelle ils seraient traités, mais à la qualité du dialogue proposé.

\section{Le traducteur partenaire}

«Faire du théâtre aujourd'hui, écrire pour la scène, s'emparer d'un texte de théâtre ou choisir des textes non théâtraux pour composer une écriture scénique est une manière de parler du monde, de celui qui nous traverse, et de se poser face à lui en tant que sujet créateur», écrit Jolivet Pignon (2015: 18). "S'emparer d'un texte» est la bonne formule pour décrire le but commun qui concerne ici à la fois le metteur en scène, le dramaturge et, bien évidemment, le traducteur, qui travaille en étroite collaboration avec eux, ainsi qu'avec les acteurs. Tout repose sur la relation de confiance qui existe entre l'ensemble des créateurs du projet et l'auteur et, dans la mesure où ce dernier a donné son accord, il n'y a pratiquement pas de limites à ce qui peut être fait. Il arrive qu'inévitablement, à un moment donné, le résultat cesse d'être une traduction pour devenir une adaptation ou une dramaturgie (dans la mesure où elle est, in fine, l'œuvre du dramaturge). Le processus de travail est, de ce point de vue, passionnant à observer.

Voici comment Wajdi Mouawad, auteur et dramaturge ${ }^{4}$, décrit sa collaboration - et son amitié - avec le metteur en scène Krzysztof Warlikowski ${ }^{5}$ :

La première fois, c'était en 2009, un coup de fil pour me demander si je voulais bien traduire en français Un tramway nommé désir de Tennessee Williams pour sa mise en scène à l'Odéon. Il avait besoin d'un texte éclaté, proche du canevas, pour y cheminer à son aise [...]. Il me rappelle un matin de septembre 2014 [...]. La demande était simple. Krzysztof préparait pour le Théâtre de l'Odéon un spectacle composé des textes de quatre auteurs qui ont écrit autour du personnage de Phèdre: Euripide, Sénèque, Sarah Kane et J. M. Coetzee. «Les pièces d'Euripide et de Sénèque, me dit-il, me servent pour réaliser la première partie [...]. Est-ce que tu pourrais revoir la traduction qui a été faite à partir du grec et latin? J'aurais besoin que ce soit plus poétique, plus moderne aussi, peux-tu y jeter un coup d'œil ?...» J'ai accepté sans hésiter, d'autant qu'il ne s'agissait que de procéder à une vérification. J’aurais dû me méfier [...]. Au fil des jours, Krzysztof m’a demandé d'inventer des répliques, des idées et des intentions absentes des textes originaux. Nous dérivions. Je recevais des indications nouvelles, parfois contradictoires, sur CEnone, Phèdre et Hippolyte, une invitation à regarder tel film de Jarmusch, tel autre d'Antonioni, de sorte qu'il devenait impossible de rester fidèle aux auteurs tout en satisfaisant les intuitions de Krzysztof. Nous avons beaucoup échangé là-dessus. (Mouawad 2016: 10-11)

À l'évidence, s'il avait été question d'auteurs vivants ou d'ayants droit, un tel travail n'aurait pas pu se faire sans leur accord, plus ou moins facile à obtenir. Les héritiers de Tennessee Williams n'avaient d'ailleurs pas accepté la démarche concernant Un tramway nommé désir, ce qui explique que le spectacle a été présenté sous le titre Un Tramway, avec la précision qu'il s'agissait d'une œuvre «d'après » et non pas «de» Williams. Quant au spectacle Phèdre(s), c'est finalement Mouawad qui a signé le texte. Voici un théâtre où, une fois que l'on a donné son accord, «le reste est travail, acharnement, exigence» pour chacun des participants au spectacle, y compris le traducteur. Il ne convient pas à tout le monde, mais il est clair qu'il laisse un espace remarquable à la création. 


\section{De la versatilité du rôle du traducteur}

Le rôle crucial d'une collaboration intense et d'une relation de confiance entre un(e) auteur(e)/metteur(e) en scène et un(e) traducteur(-trice) se trouve confirmé par beaucoup d'autres exemples, comme celui d'Angélica Liddell ${ }^{6}$ et de sa traductrice en français, Christilla Vasserot:

Nous avons des liens très proches [...]. Je traduis souvent au fur et à mesure qu'elle écrit, parce qu'elle écrit pour la scène et qu'on a besoin assez vite d'une version française pour le sur-titrage. Donc je suis de près le processus de création. Je l'accompagne pas à pas [...]. J'assiste aux répétitions [...]. Je modifie les versions en temps direct. L'accès aux variantes successives me donne énormément d'informations pour la restitution en français. Voir que tel terme a remplacé tel autre, et qu'Angélica a tourné ici, autour du sens, là, autour du rythme, m'aide à moduler mes choix de traduction. (Vasserot 2015:9)

Cette dernière ajoute «qu'il y a différents formats de traduction: un pour le surtitrage, un pour le livre et la constitution du répertoire» (2015: 9). Nous aborderons plus loin des problèmes propres au sur-titrage. Il s'avère pourtant que le format livresque n'a pas disparu, tant s'en faut, dans ce théâtre qui n'est plus un théâtre de répertoire. Bien sûr, les textes sont toujours publiés, et ils présentent, souvent, une qualité littéraire considérable. Cependant, comment savoir s'ils peuvent être lus (et être compris) indépendamment de leurs réalisations sur le plateau? Carl Djerassi, dans son livre La Science au théâtre, qui traite des «pièces faites pour être lues et non jouées", avance une hypothèse intéressante: il affirme que "voir sa pièce lue et publiée, plutôt que jouée très occasionnellement, peut et doit constituer une motivation suffisante pour un nombre non négligeable d'auteurs» (2014: 8). La raison en est que «la grande majorité des pièces, qu’elles soient classiques ou modernes [...], sont très peu jouées» (2014: 7). Djerassi, chimiste de son métier et auteur de théâtre par passion, exprime ici un point de vue que seule une personne extérieure à une vie théâtrale active peut exprimer. Certes, la plupart des pièces écrites ne sont que peu jouées (et peu publiées - une réalisation scénique étant généralement une condition pour la publication d'une pièce). Mais le propre de l'écriture théâtrale reste fondamentalement de devenir paroles. C'est d'autant plus vrai aujourd'hui que la production du texte et la matérialité du plateau ne sont plus dissociables, à tel point qu'on parle maintenant d'«écrivains de plateau», pour utiliser le terme forgé par Bruno Tackels (2005). Avec ce genre d'écriture, une publication, bien qu'importante, ne sera jamais qu'un but secondaire.

\section{La traduction à l'envers ou le traducteur pleinement créateur}

«La scène invente le texte en tant que concrétion scénique d'un travail théâtral pouvant donner lieu à une publication séparée, qui ne rendra compte alors que d'une partie de l'événement théâtral», dit Danan (2015: 14). Ainsi, Le Cycle des résurrections de Liddell, dans la traduction de Vasserot, a été publié par Les Solitaires Intempestifs en 2014. La pièce Épitre de saint Paul aux Corinthiens n'y occupe que six pages. D’après Vasserot, ceci est dû au fait que le cycle

était conçu longtemps avant la première représentation. Le processus de création allait faire bouger le texte, c'était à prévoir. Même après la première, il évolue encore. Mais 
curieusement, quand Angélica écrit, elle n'a pas forcément conscience de ce mouvement à venir: au moment où elle envoie le texte, pour elle, il est définitif... On peut dire que cette Primera carta [L'Épître de saint Paul aux Corinthiens (Primera carta...), le spectacle présenté en décembre 2015 au Théâtre de l'Odéon à Paris], est une deuxième version, qui fera probablement l'objet d'une [autre] publication. (Vasserot 2015: 9)

La pièce (si on peut utiliser ce mot plutôt inadéquat) n'a finalement pas été publiée dans son intégralité, mais sa partie centrale, «Lettre de la Reine du Calvaire au Grand Amant», a paru chez Les Solitaires Intempestifs, toujours dans la traduction de Vasserot, dans un tome intitulé Via lucis. Ainsi, rien que pour disposer d'un texte complet, il faut une transcription extrêmement attentive. Selon ma propre expérience, lorsque le Teatr Nowy de Poznań (Pologne) m’a demandé une nouvelle traduction en polonais du Charme discret de la bourgeoisie, de Jean-Claude Carrière et de Luis Buñuel, j’ai eu la chance de disposer de la version du scénario publiée en 1973 dans L'Avant Scène Cinéma, qui contenait non seulement les didascalies, mais aussi les scènes coupées lors du montage du film. Il se trouve que ces scènes ont retenu l'attention du metteur en scène, Marcin Liber, qui les a incluses dans le spectacle, ce qui ne serait pas arrivé si, au lieu d'un exemplaire de L'Avant Scène, j'avais seulement disposé du script du film.

\section{La traduction comme spectacle ou la disparition de la traduction}

Qu'en est-il de la traduction incorporée directement dans un spectacle? Évidemment, si le texte n'est pas figé, il peut difficilement être traduit avant la représentation. Et, parfois, il n'y a pas de traduction du tout: elle ne semble pas nécessaire. Ce seuil nouveau pose la question du déplacement du transfert du sens, voire, donc, de sa nécessité.

La situation de la traduction dans le spectacle ou quasiment sur scène se rapproche de la situation pragmatique de l'interprète; et les risques, pour le traducteur comme pour la traduction, sont sans doute plus grands que dans une situation ordinaire. Et pourtant, on ne parlera sans doute pas d'«interprétation" au sens où on l'entend quand il s'agit d'interprétation simultanée ou consécutive. La raison en est, bien entendu, le jeu scénique et l'enjeu de la théâtralité ainsi que la présence des spectateurs.

Personnellement, je partage l'avis d'un homme de théâtre français avec lequel j'ai eu l'occasion de voir un spectacle polonais non sur-titré. Non seulement la situation ne le gênait absolument pas, mais il appréciait l'absence de toute traduction. Il a déclaré n'avoir aucun problème à voir un spectacle sans en comprendre la langue, car il se concentrait, dans ce cas, sur d'autres aspects de la représentation. C'était pour lui un véritable critère: si un spectacle pouvait l'intéresser sans qu'il sache ce qu'on y dit, cela prouvait à ses yeux que c'était, effectivement, du bon théâtre, fonctionnant sur de multiples plans et non seulement sur le plan verbal.

Une traduction sur le vif peut être difficile à mettre en place dans le cas de spectacles en plein air, par exemple, ou de spectacles multilingues. Il y a également des spectacles choraux, où les sonorités précises - proches du chant - servent à faire passer une énergie. Là aussi, se pose la question de la nécessité du sens. Peut-on parler d'une poétique qui serait au seuil du sens, donc au seuil de la traduction? D’une expérience esthétique, au théâtre, d’où serait exclue la nécessité de la traduction? 
Marqué par l'héritage de Jerzy Grotowski, héritage expérimental et en même temps profondément ancré dans les traditions anciennes, ce type de théâtre ressemble souvent à un rituel. Le théâtre Gardzienice ${ }^{7}$, dirigé par Włodzimierz Staniewski, en est un exemple. Un renoncement à la traduction peut aussi venir d'une motivation totalement différente: introduire un effet de distanciation... Quand, à la Volksbühne ${ }^{8}$ de Berlin, Jeanne Balibar lance une réplique ou un petit monologue en français au milieu d'un spectacle de sept heures qu'elle joue en allemand, rien n'est traduit, parce que le sens des mots qu'elle prononce n'a ici qu'une valeur ajoutée. Ce qui est essentiel, par contre, c'est le fait de briser l'illusion qui a pu se créer grâce à un jeu magistral. En effet, ce type de théâtre veut rappeler aux spectateurs qu'ils ne sont pas là pour se laisser séduire par un univers imaginaire, mais qu'ils ont devant eux, non pas un personnage (certes, impressionnant), mais bien une personne réelle, une comédienne française, travaillant à cet instant même sur la scène de la Volksbühne, place Rosa Luxembourg.

Un autre cas de figure est celui des improvisations. Prenons l'exemple d'un spectacle comme Regarde le lustre et articule, des Chiens de Navarre, où est reconstitué le dispositif d'une lecture à la table pour lire une pièce qui n'existe pas. Les comédiens ont devant eux des pages blanches qu'on les voit lire, alors qu'ils inventent la pièce dans une improvisation totale et non préparée. Afin de ne pas se répéter, ils se font accompagner tous les soirs d'un invité différent.

Le problème de la traduction se pose, bien sûr, lorsque le spectacle entreprend une tournée internationale. Différentes solutions peuvent être envisagées; ainsi, certaines scènes improvisées de Factory 2, de Krystian Lupa ${ }^{9}$, n'ont pas été sur-titrées lors de représentations du spectacle en dehors de Pologne, reposant ainsi sur la seule force d'interprétation des acteurs. D’autres scènes, improvisées en Pologne, ont été fixées et traduites lors de représentations devant les publics non polonophones.

Pourtant, même dans le cas d'improvisation totale, l'impossibilité de traduction peut s'avérer relative. On pourrait imaginer la participation d'interprètes, donnant la reconstitution plus au moins fidèle ou bien dialoguant avec ce qui se dit sur scène et rajoutant ainsi une couche textuelle supplémentaire au spectacle. En effet, le traducteur peut carrément se trouver in presentia sur le plateau. Dans Disabled Theater, de Jérôme Bel, les propos des comédiens - atteints de différents handicaps mentaux - sont traduits par une interprète présente à côté d'eux. Les comédiens n'incarnent pas des personnages mais parlent d'eux-mêmes, se racontent, et n'hésitent pas à s'écarter de la partition s'ils en ont envie. L'interprétariat est ainsi incorporé au spectacle, et la traductrice devient comédienne - car ce qu'elle fait n'est rien de moins que de jouer un rôle. Pour ce spectacle, la traduction se fait d'ailleurs à plusieurs niveaux. L'interprète traduit de l'allemand (le spectacle étant réalisé en Suisse alémanique) vers la langue du public non germanophone quand c'est le cas. En même temps, elle facilite la communication en ce qui concerne l'énonciation des comédiens, voire leur prononciation. Ainsi, le spectacle interroge la place des personnes handicapées dans la société, tout comme le statut de celui ou de celle qui les traduit.

\section{Le sur-titrage, ultime manifestation de la traduction?}

Les sur-titres, quant à eux, peuvent également être davantage qu'une simple traduction, et faire partie intégrante d'un spectacle. Bien souvent, ils ne sont pas affichés 
sur un écran au-dessus ou à côté de la scène, mais au fond du plateau. Ceci est censé faciliter leur lecture, tout en inscrivant le texte dans les décors. Le metteur en scène qui est allé au bout de cette logique est Jan Klata, avec la pièce de Shakespeare, Titus Andronicus. Dans cette coproduction du Teatr Polski de Wrocław et du Staatsschauspiel de Dresde, les comédiens polonais et allemands jouent chacun dans leur langue respective. Les sur-titres ne cessent d'apparaître, de passer à des rythmes différents, de surgir comme des pop ups - sur les écrans, sur les murs, sur les éléments des décors, en différentes polices d'écriture de taille différente, faisant penser tantôt à un titre de journal, tantôt à des slogans graffés sur les murs des villes... Il ne fait aucun doute qu'il s'agit ici d'un choix artistique et qu'aussi bien le texte affiché que sa forme visuelle sont essentiels au spectacle.

La première règle reste pourtant qu' «il ne faut surtout pas entraver la vision du spectacle», comme le dit Vasserot (2015: 9). Le sur-titrage, d'une manière générale, «est toujours un travail de synthèse» et, "pour aller à l'essentiel, on doit faire des choix, qui sont souvent des crève-cœur». Vasserot cite notamment un fragment de sa traduction publiée (2011), laquelle «illustre la difficulté du sur-titrage chez Liddell, quand couper, synthétiser revient à trahir le texte fondé, lui, sur la répétition d'un même rythme, la variation sur un même thème» (2015: 9):

Je suis une poulpe vaseuse à l'intérieur d'un sac.

Je suis un homme attaché à un bûcher.

Mes propres habits m'ont en horreur.

Mon âme est dégoûtée par ma vie.

Je suis un onagre dans le désert.

Je suis l'effet d'une purge.

Je suis une glaire épaisse.

Ma solitude est gothique.

Mes omoplates sont des volcans.

Mes os saillants pourraient couper du bois.

Et en présence des hommes, j’aboie. (Liddell 2011: 12-13)

Dans L'Année de Richard, Liddell prononce ce passage à toute vitesse, comme dans une transe. C'est une gageure que de la suivre dans sa parole, et davantage encore de lire les sur-titres. Vasserot relève le défi à sa façon: «Il faut sur-titrer en partant d'un principe: on n'y arrivera pas. C'est la seule façon d'y arriver», conclut-elle.

Le bon déroulement du spectacle sur-titré demande bien entendu un travail commun entre le traducteur et le metteur en scène, qui décident ensemble de ce qui va apparaître et à quel moment. C'est un travail très précis, qui concerne également les acteurs et peut impliquer des modifications dans leur jeu. Le rôle du sur-titreur est également primordial, et d'ailleurs il n'est pas rare qu'il soit accompagné par le traducteur ou un assistant.

La réalité est pourtant très éloignée de l'idéal. Toute personne fréquentant des festivals internationaux ou des spectacles en tournée est consciente de problèmes récurrents, de traductions approximatives ou même erronées, de sur-titres mal synchronisés (ou manquants), réalisés visiblement par des personnes qui ne connaissent pas le spectacle. Les metteurs en scène ont parfois tendance à minimiser ces problèmes qui risquent néanmoins de perturber la communication avec le public d'une façon significative. Il reste alors à croire au pouvoir du théâtre, qui s'avère plus fort que tout. 


\section{Conclusion}

Le rôle du traducteur dans le théâtre peut aller de l'absence (choisie ou non), donc de l'invisibilité, jusqu'à la présence sur scène. Dans tous les cas où il est présent, le traducteur doit faire preuve d'une disponibilité et d'une réactivité particulières, mais surtout d'une grande complicité avec le metteur en scène. Souvent, ce dernier fait appel à «son» traducteur, comme il s'adresse à "son » dramaturge. J'oserais dire que dans ce genre de collaboration, le seul professionnalisme n'est pas suffisant. Il faut bien connaître la démarche du metteur en scène et y adhérer, car il est difficile de participer à un tel processus sans partager les objectifs du metteur en scène et adhérer à la sensibilité qui l'anime. Dans ce théâtre de liberté et de créativité, les personnes impliquées travaillent de concert pour un même but.

Une connaissance des coulisses du théâtre et l'habitude des répétitions permettent au traducteur de trouver un ton juste et des formulations qui «fonctionnent» dans la bouche d'un acteur. Ces dernières doivent passer l'épreuve d'être dites et redites, parfois chuchotées, parfois criées devant un public qui peut compter des milliers de personnes, ou bien se trouver dans une proximité relevant de l'intime. On observe que ceux qui traduisent le théâtre, à défaut d'être des metteurs en scène, des acteurs ou des auteurs/dramaturges eux-mêmes, sont, généralement, des spectateurs avides, souvent des critiques ou d'autres spécialistes du domaine. Une expérience personnelle, des heures passées à voir les artistes s'approprier le texte en le décortiquant, en le retournant en bouche afin de voir ce qui marche ou non, voici ce qui fait la sensibilité d'un traducteur et lui permet de remplir les attentes du metteur en scène et de servir le spectacle.

Mon propre travail sur les textes, très complexes, de Paweł Demirski n’aurait pas été envisageable si je n'avais pas, pendant des années, assisté à leurs réalisations scéniques. Demirski, l'auteur de théâtre le plus marquant de ces dernières années en Pologne, travaille en duo avec la metteure en scène Monika Strzępka. Les textes sont écrits au fur et à mesure des répétitions, pour des acteurs précis, et semblent complètement indissociables des mises en scène de Strzępka. Il m’aurait été quasiment impossible d'en déchiffrer tous les sens sans avoir vu les expressions, avoir entendu les tons et les intentions dans les voix des acteurs. "La scène invente la représentation, dans un processus de "rhapsodisation généralisée", où se mêlent, se répondent, s'interpénètrent fragments textuels, sons, musiques, images...", écrit Danan (2015: 14). Ce «texte-là, aucun livre ne pourra en conserver autre chose qu'une trace partielle et éloignée» (Danan 2015: 14).

Aucun livre ni aucune traduction... N'avons-nous tout de même pas vu que la traduction, comme le théâtre lui-même, dépasse le simple travail sur le texte? Paradoxalement, c'est la création collective d'une œuvre qui rend à la traduction toute sa dimension créative, et non le processus solitaire de l'écriture. Mais dans quelles limites pourra-t-elle s'exercer comme transfert de sens ou reflet de l'ouvre originale si elle devient partie intégrante d'une œuvre multiple? Sans doute même déborde-ton le cadre de la stricte traduction pour emprunter véritablement, et pragmatiquement, un chemin vers une création totale comme si tous les corps de métier se conjuguaient pour construire une ouvre. 


\section{NOTES}

1. Henri Gouhier (1898-1994): philosophe et critique dramatique français d'inspiration chrétienne.

2. Le collectif LFK s'est construit depuis Marseille au début des années 1990, répondant à l'invitation de Jean Michel Bruyère. Il est constitué d'une équipe de base de 10 professionnels et régulièrement renforcée de 14 autres. L'équipe de LFK respecte la parité hommes-femmes. Elle fait se croiser plusieurs générations - un tiers a moins de 25 ans - et 12 nationalités en provenance de 5 grandes régions du monde. Il conçoit la création contemporaine en tant qu'acte social et les actes sociaux en tant que créations contemporaines, www.lfks.net/en/content/les-fondamentaux-de-lfk> consulté le $1^{\text {er }}$ juin 2017.

3. Notes personnelles de l'auteure.

4. Wajdi Mouawad, né en 1968: metteur en scène, auteur, comédien, directeur artistique, plasticien et cinéaste libano-canadien (québécois). Il dirige, depuis avril 2016, Le Théâtre national de la Colline à Paris.

5. Krzysztof Warlikowski, né en 1962, est un metteur en scène polonais de théâtre et d'opéras. Créateur et directeur du centre culturel international Nowy Teatr à Varsovie.

6. Angélica Liddell, née en 1966: artiste, metteure en scène, auteure et interprète espagnole. Elle a créé et dirige la compagnie Atra Bilis Teatro à Madrid. Pour le public canadien, elle a présenté son spectacle Todo el cielo sobre la tierra (El síndrome de Wendy) à Montréal lors de l'édition 2014 du Festival TransAmériques.

7. Le Centre européen des pratiques théâtrales Gardzienice, fondé en 1977 par Włodzimierz Staniewski, est un centre expérimental de recherches et de réalisations théâtrales, situé à Gardzienice en Pologne.

8. La Volksbühne Berlin (actuellement Volksbühne am Rosa-Luxemburg-Platz) est un théâtre de Berlin (das Volk signifie: le peuple, die Bühne: la scène, le théâtre). Sa création en 1890 s'est appuyée sur un mouvement parti des couches populaires. Dans la tradition du théâtre politique lancée par Erwin Piscator, la Volksbühne s'engage dans les débats de la cité. Sous la direction de Frank Castorf, nommé en 1992, elle est devenue une référence pour le théâtre allemand et européen. Aux côtés de Castorf se sont produits des metteurs en scène comme Christoph Marthaler, Christoph Schlingensief et René Pollesch.

9. Krystian Lupa, né en 1943: metteur en scène, réalisateur, scénariste, auteur, théoricien de théâtre et pédagogue polonais.

\section{RÉFÉRENCES}

Danan, Joseph (2015): Un art à mille temps. Préface. In: Rafaëlle Jolivet Pignon. La Représentation rhapsodique. Quand la scène invente le texte. Paris: L'Entretemps.

Djerassi, Carl (2014): La Science au théâtre. (Traduit par Liliane BoIs-Simon) Paris: Presses des Mines - Transvalor.

Gouhier, Henri (1989): Le Théâtre et les arts à deux temps. Paris: Flammarion.

Jolivet Pignon, Rafaëlle (2015): La Représentation rhapsodique. Quand la scène invente le texte. Paris: L'Entretemps.

Lehmann, Hans-Thies (2002/2010): Le Théâtre postdramatique. (Traduit par Philippe-Henri LEDRU) Paris: L'Arche.

Liddell, Angélica (2006): El año de Ricardo. León: Caja España.

Liddell, Angélica (2006/2011) : L'année de Richard. (Traduit par Christilla VAsserot) Besançon: Les Solitaires Intempestifs.

Mouawad, Wajdi (2016): Une chienne. Paris: Leméac/Actes Sud-Papiers.

Tackels, Bruno (2005): Les Castellucci - Écrivains de Plateau I. Besançon: Les Solitaires Intempestifs.

VAsserot, Christilla (2015): L’Amour en toutes lettres. Lettre de l'Odéon. 16(octobre-novembre):9. 